\title{
SIMULTANEOUS OPTICAL AND UV SPECTRA OF THE TWO LMC NOVAE 1988
}

\author{
H. Drechsel and $J$. Andreae \\ Dr. Remeis Observatory Bamberg \\ Astronomical Institute of the University Erlangen-Nuernberg, F.R.G. \\ S. Starrield \\ Department of Physics and Astronomy \\ Arizona State University, Tempe, AZ, U.S.A. \\ G. Sonneborn \\ IUE Observatory, NASA Goddard Space Flight center \\ Greenbelt, $M D, U . S . A$.
}

\section{ABSTRACT}

The two classical novae of 1988 in the LMC are the first extragalactic novae, which have been studied in the UV range. IUE spectra were taken under the Nova Target of Opportunity program, and quasi-simultaneous optical spectra were obtained by the authors. The spectral development in the UV and viaual ranges is discussed for both objects.

\section{NOVA IMC 1988 No.1}

The outburat of the classical nova N LMC 1988 No.1 was discovered on March 21, 1988. The first IUE (SWP+LWP) spectra were taken only a few days later between March 25-30, 1988. An extensive set of UV spectra covers the subsequent outburst phases until the late nebular stage in May 1989. Quasi-simultaneous optical spectra in the range $380-710 \mathrm{~nm}$ were obtained during the early decline with the ESO $1.52 \mathrm{~m}+\mathrm{B} \& \mathrm{C}+\mathrm{CCD}$ on March 26-29. The overall spectral appearance and development suggest no evident deviations from the behavior of similar galactic novae, and are especially reminiscent of Nova V1668 Cyg (1978). The chemical composition of the ejecta is compatible with a co-type white dwarf.

The UV spectra of the early outburst stages show emissions of C I-II, Si I-II, Fe I-II, Al II, Ni II, Mg II. Mg II (280) has a marked P Cyg profile with an absorption edge at about $-2000 \mathrm{~km} / \mathrm{s}$. Until the end of April, a strenthening of the far UV continuum was observed and accompanied by an increase in ionization stage. The resonance fluorescence line of 0 I (130.4) exhibits drastic variations during the early phases: it is absent shortly after outburst, becomes the strongest UV line in late April and May, and then gradually decreases and vanishes in July 1988. By the end of May, a wide range of ionization potential is present ( $\mathrm{I}-\mathrm{N} \mathrm{V})$. Intercombination lines like C III] 190.9, N II $]$ 175.0, N IV] 148.6 , O IIT + C I T 232.6 increase in strength. A late nebular spectrum of May 8, 1989, contains only high ionization, intercombination and forbidden lines.

The strongest lines in the early outburst visual spectra are the Balmer series lines, He I (587.6) and Fe II lines (e.g. at 492, 502, 518 nm), superimposed on a relatively strong continuum increasing towards the blue edge of the visual range. The lines have distinct $P$ cyg profiles, with absorption velocities of about -1000 to $-1500 \mathrm{~km} / \mathrm{s}$. The FWHM of the emissions amounts to about $1200 \mathrm{~km} / \mathrm{s}$. 
NOVA LMC $1988 \quad$ NO.2

This object was discovered on oct. 12, 1988. It was also declaredas IUE Target of opportunity, and UV spectra werefirst taken on $0 \mathrm{ct}$. 14 and 21 , and at several later dates until May 25, 1989. Optical CCD spectra were obtained between oct. 30 and Nov. 26, 1988, in the range of 380-860 $\mathrm{nm}$ with the ESO $1.52 \mathrm{~m}$ and $2.2 \mathrm{~m}$ telescopes. The decline rate of this fast nova is larger by a factor of 4-5, and the expansion velocities by a factor of 2-3 compared with N LMC 1988 No.1. The chemical abundances of the ejecta suggest an O-Ne-Mg white dwarfas progenitor of this fast neon nova.

In the first UV spectra, Mg II, Fe II and Si II Iines with distinct P Cyg structure are prominent. By the end of october, the strongest lines are Si II $(126.5,130.8)$. Also numerous other lines of Si II, $\mathrm{Ni} I$, A1 II-III, and Fe III are present. Carbon lines are practicaliy absent or very weak. SWP and LWP spectra taken in the late nebular stage by Gonzalez-Riestra and Cassatella on May 21 and 25, 1989, show $\mathrm{NV}(124.0), \mathrm{NV}(148.6), \mathrm{Mg}$ V $\mathrm{MgII}(280)$ and features at 158.4 and $264.3 \mathrm{~nm}$ as strongest ines.

The earliest optical spectroscopy is reported by Ruiz and Heathcote (IAU Circ. 4664), who took spectra between 360 and $690 \mathrm{~nm}$ with the CTIO $4 \mathrm{~m}$ telescope on oct. 15: superimposed on a strong blue continum are broad Balmer emission lines with marked P Cyg profiles as well as Fe II lines at $492,502,518 \mathrm{~nm}$. The FWHM was about $3100 \mathrm{~km} / \mathrm{s}$, the absorption edges extended to $-3650 \mathrm{~km} / \mathrm{s}$. Our first optical spectra were obtained between oct. 30 and Nov. 1, 1988, between 370 and $540 \mathrm{~nm}$. The strongest features are still the Balmer lines. The profiles are of P Cyg type, with high velocity absorption components extending to about $-4000 \mathrm{~km} / \mathrm{s}$. The broad emissions have central peaks with a width of about $800 \mathrm{~km} / \mathrm{s}$. By the middle of November, the ratio of emission/absorptionstrength had increased so much that only marginal absorption components had remained. The FWHM of the emissions amounts to about $4000 \mathrm{~km} / \mathrm{s}$. The structure of the emissions is complicated. The spectra of the period Nov. 19-26 show a further increase of the emission strengths. The FWH of $\mathrm{H}$ beta amounts to $4200 \mathrm{~km} / \mathrm{s}$. The emissions have several distinct sharp components suggesting clumping and a filamentary structure of the envelope. The strongest line in the covered range is now the blend of N III $(464.0)$ and $\mathrm{He}$ II $(468.6)$.

\section{CONCLUSIONS}

For the first time, simultaneous UV and optical spectra are available for extragalactic classical novae. A quick-look analysis suggests that N LMC 1988 No.1 exhibits no extraordinary properties compared with similar galactic novae. It belongs to the Co-type objects. N IMC 1988 No.2 is of a faster speed class, has larger expansion velocities, and the chemical abundance anomalies suggest it to be another "neon nova". The data presented here might be useful to assist in a project initiated by stryker et al.: a new method is being developed for element abundance determination, which uses expanding stellar atmosphere calculations for supernova and nova shells at early outburst stages. Such a method would be completely independent from the usually applied nebular analysis techniques. 